\title{
LIDERAZGO EDUCATIVO Y FORMACIÓN CIUDADANA: VISIONES Y PRÁCTICAS DE LOS ACTORES $^{1}$
}

\author{
Camila Jara², Macarena Sánchez ${ }^{3}$ y Cristián Cox $^{4}$
}

\begin{abstract}
RESUMEN
Este estudio indaga en la relación entre el liderazgo escolar y la formación ciudadana en el contexto escolar luego de la ley que en 2016 redefinió este ámbito de la experiencia escolar. Para ello, se analizaron datos primarios cualitativos provenientes de una muestra de establecimientos educacionales con un alto indicador de participación y formación ciudadana medido por la Agencia de Calidad de la Educación, con el propósito de profundizar en los estilos y prácticas de liderazgo que caracterizan la educación ciudadana en estos establecimientos. Los resultados muestran que el liderazgo educativo tiene un papel relevante, aunque indirecto, en la formación ciudadana a través del desarrollo de identidad y sentido de pertenencia. Por otra parte, se identifica que la implementación de la Ley de Formación Ciudadana no ha alterado aún el reduccionismo de la educación ciudadana a la asignatura y al docente de Historia, así como la prevalencia de la formación valórica por sobre la alfabetización política. El estudio revela que la implementación del Plan de Formación Ciudadana exigido por la mencionada ley tiene, por ahora, leves consecuencias sobre prácticas preexistentes.
\end{abstract}

Conceptos clave: formación ciudadana, liderazgo educativo, socialización política.

\section{EDUCATIONAL LEADERSHIP AND CITIZENSHIP EDUCATION: ACTORS' VISIONS AND PRACTICES}

\section{ABSTRACT}

This article focuses on the relationship between school leadership and citizenship education in Chile, after the 2016 Citizenship Education Law, which reframed the scope of school experience in this field. Qualitative primary data from a sample of schools with a high indicator of Citizen Participation and Formation measured by the official Education Quality Agency, is analysed in order to describe and examine leadership styles and practices. Results from interviews with school head-masters and head-mistresses, teachers and students, show that educational leadership has a relevant although indirect role in citizenship education, especially through a sense of identity

1 El presente artículo forma parte de los resultados del estudio Liderazgo educativo y desarrollo de competencias ciudadanas en el contexto escolar, realizado en 2018. El proyecto contó con el apoyo del Ministerio de Educación de Chile, Mineduc, mediante el financiamiento del Centro de Desarrollo del Liderazgo Educativo (CEDLE), que integran varias universidades con la coordinación de la Universidad Diego Portales.

2 Universidad Diego Portales, Santiago, Chile. Contacto: camila.jarai@mail.udp.cl

3 Universidad Diego Portales, Santiago, Chile. Contacto: macasanchezb@gmail.com

4 Universidad Diego Portales, Santiago, Chile. Contacto: cristian.cox@udp.cl 
and a sense of institutional belonging. On the other hand, the implementation of the Citizenship Education Law has not, as yet, changed the reductionism of the area to the subject and teacher of History, or the emphasis on values over political literacy, that characterize prevailing practices. The study reveals that the Citizenship Education Plan demanded by the law, has had only minor consequences on current school practices, but there is a promising potential in terms of greater visibility and reflection in this area.

Key concepts: citizenship education, citizenship education plan, school leadership.

\section{Introducción}

Desde hace más de una década tanto el sistema político como el campo académico han diagnosticado y ven con alarma el incremento en los niveles de desconfianza hacia las instituciones y la merma en la participación en procesos eleccionarios y en organizaciones políticas convencionales (Consejo Asesor Presidencial Contra los Conflictos de Interés, el Tráfico de Influencias y la Corrupción, 2015; Joignant, Morales \& Fuentes, 2017). La respuesta más reciente a este fenómeno es la promulgación de la Ley No 20.911 a principios de 2016, que crea un Plan de Formación Ciudadana (en adelante PFC) transversal a los niveles de enseñanza, junto con la reposición de la asignatura obligatoria de Formación Ciudadana para $3^{\circ}$ y $4^{\circ}$ año de enseñanza media.

Al explicitar la responsabilidad institucional por la formación ciudadana, la Ley No 20.911 pone al liderazgo en el centro, ya que el éxito de la implementación de esta reforma y el efectivo desarrollo de la formación ciudadana en los estudiantes se encuentran estrechamente vinculados tanto al liderazgo de una comunidad educativa como al quehacer docente. Como señala el Informe de la Comisión de Formación Ciudadana (Ministerio de Educación de Chile, Mineduc, 2004), los directores "se constituyen en actores clave para asegurar que la reflexión y las acciones necesarias para el desarrollo de la formación ciudadana efectivamente cuenten con un espacio y se desarrollen de manera adecuada" (Mineduc, 2004, p. 58). La ley de 2016, entonces, establece la conjunción liderazgo-formación ciudadana, de manera que no tiene precedentes. 
Con estos antecedentes, se vuelve pertinente observar en qué medida los líderes educativos podrían impactar en el desarrollo de las competencias ciudadanas de los estudiantes. Este trabajo busca analizar la relación entre el liderazgo educativo y la formación ciudadana en el contexto escolar, examinando las características de los liderazgos de comunidades educativas con buenos resultados en el área de formación ciudadana, con el propósito de observar y profundizar sus prácticas de liderazgo.

Con este fin, se utilizó una metodología cualitativa generando datos primarios provenientes de una muestra de diez establecimientos educacionales. Los centros educativos se seleccionaron sobre la base del indicador de participación y formación ciudadana medido por la Agencia de Calidad de la Educación (ACE), con el criterio de que los establecimientos estuvieran dentro de los mejores resultados en los diferentes territorios. En cada establecimiento se realizaron entrevistas al director o directora, a los docentes con responsabilidades en la formación ciudadana, y a estudiantes de $4^{\circ}$ año medio.

El artículo se encuentra estructurado en cuatro secciones. En la primera se realiza una revisión de los principales conceptos y de la evidencia previa respecto del liderazgo educativo y la formación ciudadana en el contexto escolar. Luego, se exponen los principales elementos de la metodología, para continuar con la tercera sección, donde se presentan los principales resultados del análisis y, finalmente, se exponen las conclusiones más significativas.

\section{Marco conceptual}

\subsection{Liderazgo educativo: lo que indica la evidencia}

Durante los últimos años, las políticas educativas del país han introducido el concepto del liderazgo como uno de los aspectos fundamentales para el mejoramiento de los aprendizajes y la experiencia educativa (Mineduc, 2015). Este énfasis se basa en la existencia de abundante evidencia en cuanto a los efectos positivos que tiene el liderazgo orientado a mejorar la docencia y el contexto escolar en los resultados de los estudiantes (Flessa, Bramwell, 
Fernández \& Weinstein, 2018; Robinson, Hohepa \& Lloyd, 2009; Vaillant, 2011).

El concepto de liderazgo educativo comprende, entre otros aspectos:

- la capacidad de crear una visión y establecer lineamientos;

- distribuir recursos estratégicamente;

- garantizar una enseñanza de calidad;

- liderar el aprendizaje y desarrollo docente;

- rediseñar la organización y asegurar un ambiente ordenado y seguro (Leithwood, Harris \& Hopkins, 2008; Robinson et al., 2009).

La influencia del liderazgo educativo se concreta mediante prácticas orientadas hacia el mejoramiento escolar. Por esto, la investigación en esta materia se centra en identificar los repertorios de prácticas comunes que inciden positivamente en los resultados escolares (Horn y Marfán, 2010; Leithwood, Sammons, Anderson \& Wahlstrom, 2004).

De esta manera, la evidencia ha enfatizado en las prácticas de liderazgo que resultan valiosas para la mejora del proceso de aprendizaje, como forma de entender el liderazgo educativo (Robinson et al., 2009). Estas prácticas tienen como principal objetivo mejorar el quehacer de los docentes, lo que se relaciona con sus creencias, valores, motivaciones, capacidades y conocimientos, así como con mejorar el contexto, las condiciones y el clima en el que trabajan (Leithwood et al., 2008).

Al respecto, la literatura acerca del liderazgo educativo coincide en que los efectos sobre los aprendizajes son principalmente indirectos (Hallinger \& Heck, 2010; Leithwood \& Jantzi, 2000). Esto quiere decir que el liderazgo incide en los resultados de los estudiantes a través de los efectos positivos que puede tener en el contexto escolar. De esta forma, al pensar en un modelo de liderazgo, es necesario considerar que existen variables mediadoras de sus efectos en el aprendizaje, es decir, que existen conectores que unen 
las prácticas del liderazgo con el mejoramiento escolar (Hallinger \& Heck, 2010).

Dentro de los aspectos en los que los líderes inciden se encuentran la cultura escolar, el clima laboral y el compromiso de los docentes, a lo que se suma el conjunto de oportunidades de aprendizaje de los estudiantes (Day et al., 2009). A su vez, los líderes pueden incidir en esta última dimensión mediante la implementación, priorización y énfasis que se le da al currículo educativo, a las prácticas docentes y a toda acción que esté orientada directamente al mejoramiento en la enseñanza y el aprendizaje, en lo que ha sido definido como el liderazgo instruccional o pedagógico (Waters, Marzano \& McNulty, 2003).

\subsection{Formación ciudadana: socialización política y desarrollo de competencias ciudadanas en el contexto escolar}

Hace más de una década el Mineduc (2004) convocó a una comisión para dar tratamiento al tema de la formación ciudadana en la experiencia escolar, debate que resurgió en 2015 luego de las recomendaciones contenidas en el Informe del Consejo Asesor Presidencial Contra Conflictos de Interés, el Tráfico de Influencias y la Corrupción (2015), que concluyó explicitando la importancia de otorgar mayor énfasis a la formación ciudadana en el sistema escolar. Con ello, a principios de 2016 se promulgó la Ley No 20.911 antes referida. Asimismo desde 2013, la ACE ha establecido un nuevo marco que incluyó un conjunto de indicadores de desarrollo personal y social (IDPS), donde la participación y formación ciudadana pasa a ser uno de los cuatro indicadores que son estudiados desde entonces ${ }^{5}$.

La formación ciudadana en la experiencia escolar es un componente fundamental del concepto más amplio de socialización política —o el conjunto de procesos a través de los cuales niños y jóvenes aprenden orientaciones y patrones de comportamiento ciudadano (Whyte, 1999)_, y ha resurgido como objeto de interés público y académico, estimulado por los cambios en la cultura

5 Completan la lista la autoestima académica y motivación escolar, el clima de convivencia escolar, y los hábitos de vida saludable (ACE, 2015a). 
política, así como por el debilitamiento de los modos tradicionales de participación política (Amnå, 2012).

En la tarea de definir la noción de ciudadanía en su proyección educativa, se debe destacar la distinción entre las dimensiones cívica y civil. La primera es entendida en relación con lo político y las instituciones, en un sentido más vertical, mientras que la segunda es atingente a la convivencia y a la relación con los otros en el espacio más inmediato, en un sentido horizontal (Cox, Bascopé, Castillo y Miranda, 2015).

En términos de la dimensión civil de la ciudadanía, la identidad y sentido de pertenencia son factores relevantes, ya que una identidad compartida es requisito de la cohesión social y facilita el involucramiento y el compromiso de los miembros de una comunidad respecto de los eventos que le atañen (Friedkin, 2004). En este sentido, Flanagan y Levine (2015) destacan los beneficios de la participación en términos de la satisfacción de la necesidad humana de pertenencia, la conexión con otros y la construcción de capital social.

Respecto de los factores que inciden en la socialización política a nivel escolar y que favorecen la disposición y el desarrollo de competencias ciudadanas, la investigación ha destacado dos perspectivas: las oportunidades de aprendizaje provistas por la educación (Bonhomme, Cox, Tham y Lira, 2015; Isac, Maslowski, Creemers \& Werf, 2014; Mardones, 2015); y la inserción de los estudiantes en un contexto democrático, de apertura a la discusión en el aula, de participación escolar y en actividades que los vinculen con la comunidad (Campbell, 2008; Castillo, Miranda, Bonhomme, Cox \& Bascopé, 2014; McDevitt \& Kiousis, 2006).

Un modelo reconocidamente influyente en la conceptualización de los contextos clave de la formación ciudadana en el ámbito escolar es el de David Kerr (2015), que en forma adaptada utilizamos en el presente estudio. El modelo distingue tres ámbitos donde la ciudadanía —en sus dimensiones cívicas y civiles, de conocimiento, habilidades y actitudes- es ofrecida en las experiencias escolares más robustas y consistentes al respecto: el ámbito del currículo o 
de los contenidos en torno a la sociedad y al sistema político, el de las relaciones de participación y representación intrainstitución escolar, y el de las relaciones entre actores de la institución escolar y su comunidad externa, local y macro.

En Chile, las oportunidades de aprendizaje relativas a la formación ciudadana contenidas en el currículo oficial han experimentado un enriquecimiento durante las últimas décadas. Hasta 1998, los contenidos estaban concentrados en una asignatura de Educación Cívica en $3^{\circ}$ y $4^{\circ}$ año medio que enfatizaba contenidos asociados al patriotismo, la familia y la Constitución (Bascopé, Cox y Lira, 2015). En el marco curricular adoptado en 1998, la asignatura es reemplazada por nuevos contenidos específicos y objetivos transversales: la formación ciudadana se distribuye en las asignaturas de Historia y Orientación (en la educación básica), e Historia y Psicología y Filosofía, en el nivel medio. Una década más tarde, se define un eje de formación ciudadana en la asignatura de Historia y Ciencias Sociales, presente en todos los niveles de enseñanza, y un eje de participación democrática en Orientación (Cox y García, 2015).

A nivel nacional, la evidencia empírica aportada por la prueba ICCS $^{6} 2016$ indica que los establecimientos con un clima abierto a la discusión en clases, así como aquellos donde los estudiantes reportan haber aprendido temas cívicos, presentan mejores resultados en conocimiento cívico (ACE, 2017). Al mismo tiempo, el análisis de la versión 2009 del estudio ICCS señala que el conocimiento y participación cívica a nivel escuela influye en las expectativas de participación electoral futura de los estudiantes (Schulz, Ainley, Fraillon, Kerr \& Losito, 2010).

\subsection{Liderazgo educativo y formación ciudadana}

El aprendizaje de la ciudadanía es influenciado decisivamente por la experiencia escolar. Sin embargo, el tema específico de la

6 International Civic and Citizenship Education Study (Estudio Internacional de Educación Cívica y Formación Ciudadana). Esta investigación es una iniciativa de la Asociación Internacional para la Evaluación del Logro Educativo (IEA). 
relación entre el liderazgo educativo y la formación ciudadana en el contexto escolar establecido por la Ley No 20.911 no ha recibido mayor atención por parte de la investigación educacional del país. La excepción se encuentra en un estudio realizado por la ACE (2016) donde se señala que en establecimientos donde los profesores perciben mayor liderazgo del equipo directivo, los indicadores de participación y formación ciudadana presentan mejores resultados.

Por su parte, la investigación realizada por Mitra, Serriere y Stoicovy (2012) respecto de la relación entre el liderazgo educativo y el desarrollo de la "voz de los estudiantes" (p. 104) —entendida como la posibilidad de que estos compartan sus opiniones acerca de los problemas escolares con administradores y profesores- resulta de gran interés. Para los autores, la voz de los estudiantes puede llevar a un aumento en el compromiso cívico, en la medida en que influye en la creencia de los jóvenes de que ellos son capaces de hacer una diferencia en sus propias vidas y en las de los demás. De esta forma, el líder de una comunidad educativa juega un rol relevante en tanto logra:

- una visión clara respecto del proyecto institucional, que es traducida en prácticas cotidianas incorporando a los profesores;

- reconocer y valorar la autonomía de los profesores para moldear este proyecto en relación con los talentos y necesidades de los estudiantes; y

- ser flexibles con los docentes y sus intereses en la implementación de iniciativas de voz estudiantil (Mitra et al., 2012).

De igual manera, Martínez, Silva, Morandé y Canales (2010) indican que las instituciones escolares que practican una gestión más horizontal, además de contar con un sentido de pertenencia al establecimiento, facilitan el desarrollo de competencias ciudadanas y democráticas por parte de los estudiantes.

\section{Metodología}

Se realizó un muestro por conveniencia, con una selección de establecimientos que garantizaran diversidad territorial y dependencia. La investigación incluyó establecimientos en las regiones 
Metropolitana, de Atacama y de La Araucanía, y de las dependencias particular pagado, particular subvencionado y municipal, en cada uno de los territorios.

Se seleccionaron establecimientos que tuvieran buenos resultados (para el año 2016 en $2^{\circ}$ año medio) en el indicador de participación y formación ciudadana que construye la ACE como parte de los indicadores de desarrollo personal y social (ACE, 2015a). Este indicador comprende tres dimensiones: participación, sentido de pertenencia y vida democrática (ACE, 2015a) y oscila entre los valores 1 y 100 , siendo este último el puntaje máximo. El propósito de utilizar este criterio fue aproximarse a los centros educativos con buenos resultados en formación ciudadana, para conocer y profundizar en las prácticas de liderazgo que permitieron la obtención de dichos resultados, esperando con ello observar buenas prácticas para la formación ciudadana. Además, se seleccionó un establecimiento con bajos resultados en este indicador, con el fin de tener un punto de comparación ${ }^{7}$.

La Tabla 1 resume la muestra de establecimientos contemplada en este estudio y sus características institucionales distintivas.

7 Es importante destacar el uso del término "punto de comparación" y no "control" para el caso seleccionado que muestra un bajo indicador de formación ciudadana, pues para constituirse en lo segundo se requeriría un mayor número de casos o establecimientos con dichas características. 
CALIDAD EN LA EDUCACIÓN n 51, diciembre 2019359

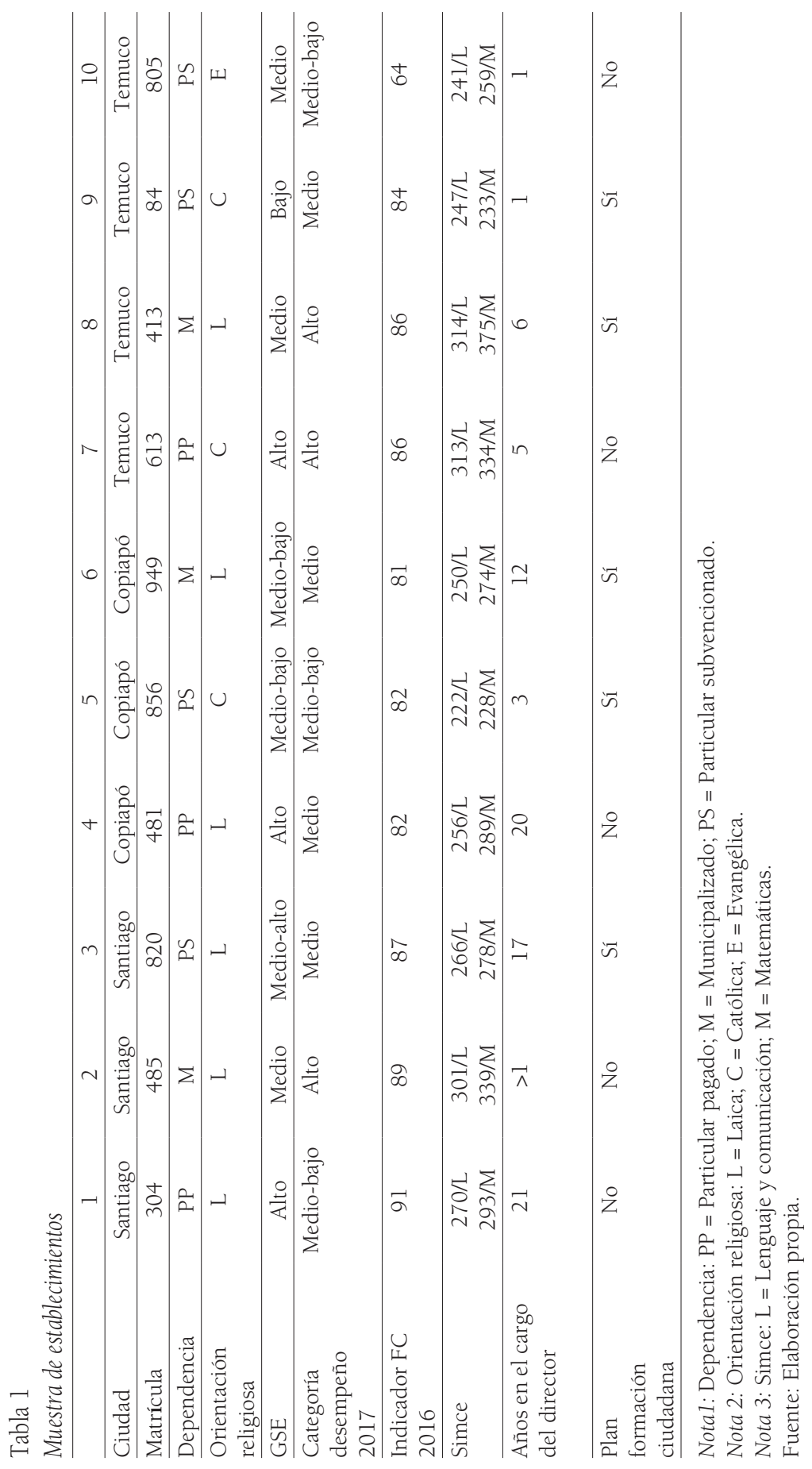


Como se aprecia en la Tabla 1, la utilización del indicador de formación y participación ciudadana del Simce como criterio de selección significó un sesgo socioeducativo de los establecimientos que, en general, comprenden grupos socioeconómicos medios o altos con buen desempeño académico.

Como otra limitación es relevante mencionar que el indicador de participación y formación ciudadana no mide conocimientos, actitudes y comportamientos, sino que considera las percepciones de los estudiantes, padres y apoderados en cuanto a las oportunidades de encuentro y los espacios de colaboración promovidos por el establecimiento; la identificación de los estudiantes con el proyecto educativo y si sienten orgullo de pertenecer a él; así como la percepción estudiantil respecto del grado en que el establecimiento fomenta el desarrollo de habilidades y actitudes necesarias para la vida en democracia (ACE, 2015a). Debido a esto, los altos resultados en el indicador de formación ciudadana podrían no necesariamente reflejar un estado destacado en términos de prácticas democráticas en el establecimiento —es decir, de una robusta implementación de la Ley $\mathrm{N}^{\mathrm{o}}$ 20.911—, ni de mayores oportunidades de aprendizaje o prácticas pedagógicas excepcionales ${ }^{8}$. Considerando esto y, a pesar de que se seleccionaron instituciones escolares con altos resultado en este indicador, no se logró identificar un conjunto de "buenas" prácticas de liderazgo para la formación ciudadana. En cambio, los hallazgos corresponden a prácticas observadas en las que se identifican tanto fortalezas como nudos problemáticos.

Una vez seleccionados los establecimientos, se entrevistó al director y a un docente con responsabilidades en la formación ciudadana. El docente fue designado por el director de acuerdo con los propósitos de esta investigación. En cada establecimiento se seleccionaron seis estudiantes de $4^{\circ}$ año medio para participar en una entrevista grupal, considerando que entre los 16 y 18 años el interés

8 Originalmente este estudio se diseñó pensando que la muestra de establecimientos con buenos resultados en términos de la formación ciudadana se haría seleccionando casos individualizados desde la prueba ICCS 2016 o de la prueba muestral de formación ciudadana que aplicó la ACE en 2017. Esto no fue posible, por lo que los criterios de factibilidad hicieron prevalecer la opción de utilizar el indicador de participación ciudadana. 
por la política y la sociedad es más alto (Amnå, Ekström, Kerr \& Stattin, 2009). Los estudiantes fueron convocados por el director o el docente, a quienes se les instruyó seleccionar un grupo con paridad de género, diversidad en cuanto a intereses, motivación y desempeño académico y, especialmente, en lo relativo a la participación política y el debate.

Se desarrollaron tres instrumentos para entrevistar a directores, docentes y estudiantes. Las preguntas fueron formuladas buscando cubrir el modelo de relaciones construido a partir de la revisión bibliográfica. Con ello, se definieron preguntas en torno a las dimensiones:

- formación ciudadana y liderazgo,

- democracia en el contexto escolar,

- oportunidades de aprendizaje de la ciudadanía y

- PFC.

Los instrumentos fueron validados mediante juicio de expertos durante los meses de mayo y junio de 2018, en tanto que entre junio y octubre de 2018 se llevó a cabo el trabajo de campo de esta investigación, periodo en el cual se realizaron 30 entrevistas: 10 a directores, 10 a docentes y 10 entrevistas grupales a estudiantes de $4^{\circ}$ año medio, conformando un total de 80 individuos entrevistados en 10 establecimientos educacionales del país.

El plan de análisis se elaboró con foco en el actor, buscando las diferencias y similitudes discursivas entre directores, profesores y estudiantes de los establecimientos entrevistados frente a la formación ciudadana. El análisis se realizó sobre la base de las entrevistas transcritas, codificando y analizando primero las categorías de análisis abordadas en las pautas y, luego sumando categorías emergentes, para así agregar valor a la información pauteada originalmente. Todo lo anterior se realizó utilizando el software Nvivo 10.

\section{Principales hallazgos}

De manera transversal fue posible apreciar una alta valoración de los directivos por la formación ciudadana. La reciente ley en torno a la temática, así como la prominencia que tienen la desafección política y 
la desconfianza en las instituciones vinculadas con el mundo político en la discusión pública de los últimos años, ha derivado en que la temática de la participación y la formación ciudadana pasen a ser un aspecto de la formación escolar ampliamente deseado. Asimismo, los datos revelan que en los establecimientos con buen desempeño en el indicador de participación y formación ciudadana (IFC), el rol del líder es indirecto, pero influyente.

\subsection{El liderazgo favorece el sentido de pertenencia e identidad de la comunidad escolar}

En las comunidades escolares estudiadas, el líder educativo es un actor clave en la articulación del entramado microcultural, generando cohesión y permitiendo la identificación de profesores y estudiantes con el establecimiento. Lo anterior va en línea con la noción de que el liderazgo que produce mejoramiento escolar es aquel capaz de crear una visión y establecer lineamientos dentro de la comunidad educativa (Leithwood et al., 2008; Robinson et al., 2009), así como también engarza con las dimensiones consideradas en el Marco para la buena dirección y el liderazgo escolar (Mineduc, 2015) acerca del liderazgo como factor necesario para la construcción de una visión estratégica compartida.

El concepto de comunidad educativa nosotros lo hemos manejado siempre y se va inculcando mucho en los chiquillos, todos los días nosotros en la mañana entregamos un mensaje a todos los chiquillos, le llamamos "los Buenos Días", y siempre es un mensaje, de unos cinco minutos, que deja alguna enseñanza. Lo que vamos siempre incentivando más, el sentido de casa, "esta también es su casa", y un sentido de pertenencia también al lugar, al espacio, a lo que tienen, ¿ya?, a los cuidados también de los espacios" (Director establecimiento particular subvencionado, Copiapó).

Si se considera que el promedio de años en el cargo directivo en la muestra de establecimientos con alto indicador de participación ciudadana es de 10 años, es posible señalar que se trata de actores con trayectoria en sus cargos. Este hecho resulta relevante en la medida en que se trata de líderes que han sido partícipes del desarrollo del proyecto educativo, gestando los valores, misiones, visiones y cultura 
escolar en la que están insertos. En este sentido, este hallazgo no revela una relación directa entre el liderazgo educativo y la formación ciudadana en su dimensión política, sino que se observa más bien un vínculo entre el liderazgo educativo y el desarrollo del proyecto educativo institucional y, mediante esta relación, un efecto sobre el desarrollo identitario y sentido de pertenencia de la comunidad, elementos que han sido destacados como relevantes para el ejercicio de la ciudadanía (Flanagan, 2003), aun cuando conciernen más a su dimensión civil que cívica.

Un elemento que sirve para apoyar este argumento es el análisis del establecimiento incluido en la muestra que, teniendo un indicador de formación y participación ciudadana bajo a nivel regional y nacional, permite observar otro tipo de relación entre el liderazgo escolar y la formación ciudadana.

En la entrevista con la directora, se reconoce un déficit de liderazgo, con problemas en la consolidación de un equipo directivo que genere identidad y que promueva un proyecto educativo compartido; ella señala que el bajo sentido de pertenencia es atribuible a la alta rotación de directivos, recalcando que los directores anteriores no ejercieron el cargo por más de dos años.

Ese fue el primer diagnóstico que nosotros hicimos al asumir, porque todo el equipo directivo es nuevo. Nos dimos cuenta que no, no teníamos una cultura de comunidad. Nosotros debemos ser una comunidad educativa, pero lamentablemente no lo somos aún, y para eso tenemos que fortalecer... y ahí nos dimos cuenta, "¿por qué no somos una comunidad educativa?, ¿qué es lo que no estamos haciendo, qué estamos haciendo mal? Nos dimos cuenta que lo que no estábamos haciendo era trabajar formación ciudadana (Directora establecimiento particular subvencionado, Temuco).

Este diagnóstico permite reconocer una relación entre el liderazgo o, en este caso, una ausencia del mismo, con un déficit identitario. A la vez, recalca la importancia de que la formación ciudadana se dé en un contexto de comunidad y con un proyecto educativo consolidado. 
En este sentido, la evidencia se relaciona con lo planteado por Mitra et al. (2012) respecto del rol del liderazgo educativo en facilitar la "voz" del estudiante. Para estos autores, una de las principales formas de influencia del equipo directivo es orquestar el desarrollo de una visión, transformándola en prácticas que se integran al funcionamiento cotidiano con la expectativa de que todos los actores escolares la incorporen en sus quehaceres. Más aún, evidencia estadística recogida recientemente desde la muestra chilena del estudio ICCS 2016, señala que la antigüedad del director en su cargo se relaciona positivamente con conocimiento cívico y con actitudes ciudadanas (Jara Ibarra, Sánchez y Cox, 2019).

\subsection{Prevalencia de la dimensión civil por sobre la dimensión cívica}

Aunque existe en el discurso de los directivos una marcada preocupación por la formación ciudadana, en la práctica se observa una tendencia a asimilarla con la formación valórica de los estudiantes, quedando la primera en una evidente desatención.

yo creo que sí estamos tratando de formar buenos ciudadanos, personas que tienen altos valores, alto respeto por los otros, por la consideración para consigo mismos también, y que quieren ser protagonistas de cambio, que es otro elemento de la ciudadanía que es sumamente necesario (Director, establecimiento particular pagado, Temuco).

Así, la alfabetización política, es decir, el proceso continuo, inacabado y progresivo de instruir o dar forma a las visiones de los jóvenes respecto del mundo político y su elección de participar o no participar en él (Dudley \& Gitelson, 2002), ha sido descuidada en favor de la dimensión civil, o de convivencia, de la formación ciudadana. Esto es, la dimensión más horizontal y que prepara para la vida en común con los cercanos, centrada en la interacción cotidiana y en la relación con el espacio próximo, en desmedro de la dimensión cívica, vertical y centrada en lo político y que prepara para la vida en común con los lejanos (Cox et al., 2015; Schulz et al., 2010). Lo señalado representa un resultado emergente, es decir, no buscado ni planificado, que tiene sus raíces en una diversidad de factores que 
tocan al currículo y sus prioridades, como la formación docente en general y la cultura del campo de la educación, los cuales apuntan en la dirección de favorecer la convivencia como foco y dejar al sistema y la participación política como trasfondo, el que muchas veces no se alcanza a distinguir o visibilizar como propósito explícito de la enseñanza. Lo señalado reafirma lo revelado en el análisis documental de los planes de formación ciudadana realizados por el PNUD (2018) que detectó un bajo énfasis en el conocimiento cívico, aspecto que concuerda con la evidencia del estancamiento consistente de los resultados de aprendizaje de los estudiantes chilenos en esta materia, evaluados por los estudios internacionales de la IEA CIVED-1999 e ICCS-2009 y 2016 (ACE, 2017). Lo constatado en los PFC se replica en los discursos y perspectivas de los directores, que al referirse a la temática de la ciudadanía tienden a equiparar al ciudadano con la persona, mientras que destacan por su ausencia términos como el voto, los derechos, la democracia, las instituciones políticas y los deberes relativos a la dimensión cívica de la ciudadanía.

Esta invisibilidad de los contenidos acerca de la política en la visión de los directivos encuentra un cierto correlato en la prescripción curricular nacional vigente entre fines de 1990 y 2013, cuando se privilegió la dimensión de convivencia democrática, y los contenidos típicos de la educación cívica tradicional centrada en las instituciones políticas, el Estado de derecho y la Constitución (Comisión Formación Ciudadana, 2004; Cox, 2003; Mardones, Cox, Farías y García, 2014) se diluyeron en una medida importante. Asimismo, este sesgo no solo se detecta a nivel nacional, sino que regionalmente también ocurre, tal como lo demostró un análisis comparado de seis currículos latinoamericanos que revela que dos tercios de los contenidos en educación ciudadana se refieren a la dimensión convivencia, y solo un tercio a la dimensión política o a la participación en política formal (Cox, Bascopé, Castillo, Miranda y Bonhomme, 2014).

Este desequilibrio a favor de la dimensión civil y la formación valórica se observa también en el discurso de los estudiantes, quienes destacan la formación en valores como predominante en las oportunidades vividas en torno a la ciudadanía. 
Entrevistadora: ¿Tienen, reciben educación ciudadana?

Estudiante 1: Directamente, yo creo que no.

Estudiante 2: No. Pero, quizás, por debajo.

Entrevistadora: ¿Cómo por debajo?

Estudiante 2: Como... por así decirlo...

Entrevistadora: ¿Por el director?

Estudiante 2: Claro, el director, es que yo creo que él... o sea, siempre va por la familia...

Pero así como ciudadanos, como formar de ciudadano, yo creo que sí el colegio te forma como ciudadano respetuoso, te inculca valores cristianos igual... si por ahí va la pregunta. Pero la parte política, ahí el colegio directamente no (Estudiantes establecimiento particular pagado, Temuco).

En forma predominante, los estudiantes perciben la ciudadanía como un constructo lejano, vinculado principalmente con la vida adulta, ya sea insertos en el mercado laboral o a través del derecho a sufragio después de los 18 años, es decir, se evidencia ajena a su quehacer y formación escolar.

\subsection{Liderazgo y autonomía docente}

En el discurso de los directivos existe una tendencia a describir sus funciones en relación con la formación ciudadana como cercanas al liderazgo pedagógico. Esto, pues señalan que ejercen una supervisión de las tareas de instrucción asumiendo funciones que contribuyen al aprendizaje de los estudiantes (Marks \& Printy, 2003).

Tenemos un profesor a cargo [de la formación ciudadana], y él está a cargo de todo el proceso, él nos está retroalimentando, y está en contacto siempre con el equipo de gestión, que somos cuatro personas, también lo tenemos siempre en carpeta. Yo siempre digo — puede ser por mi formación - yo siempre digo "oye, pero a mí me gusta que [los contenidos] sean más explícitos o más acotados" (Director establecimiento municipal, Temuco).

La evidencia señala que esta práctica, que enfatiza la independencia de los docentes para moldear su enseñanza y alinearse con las necesidades de los estudiantes, sería positiva o eficiente para 
la formación ciudadana (Mitra et al., 2012). Sin embargo, esta visión no converge necesariamente con lo que se observó en el discurso de algunos docentes encargados de la formación ciudadana.

Los hallazgos demuestran la concentración de responsabilidades de formación ciudadana casi exclusivamente en los profesores de Historia y Geografía, quedando estas temáticas relegadas a los contenidos revisados y provistos en las clases de esta asignatura. Lo anterior revela la soledad en la que se encuentra el docente encargado de estos contenidos, situación que es definida en algunos casos como un obstáculo o un elemento negativo, mientras que para otros, más alineados con la visión de los directivos, es experimentada como autonomía y confianza por parte del equipo directivo en la realización de sus labores. En el último caso, los profesores destacan positivamente que los directores proveen condiciones favorables para su trabajo educativo y el liderazgo es percibido efectivamente como cercano al pedagógico.

Desde Subdirección y Dirección hay mucho apoyo. La mayoría [en relación con] proyectos, proyectos que tenemos nosotros. Por ejemplo, la profe de tecnología creó un departamento, una comisión de medioambiente, y se le aprobó, creo que se le pagan unas horas por eso. Tienen el objetivo de reformular nuestra visión, frente a, por ejemplo, el tratamiento de residuos y dentro de eso hizo una jornada (...) eran talleres donde tú podías hacer cosas por ti mismo (Docente establecimiento particular subvencionado, Santiago).

Por el contrario, esta concentración de responsabilidades y soledad en la tarea de formar ciudadanos es percibida por otros docentes como un obstáculo. Asimismo, observan que el desarrollo de esta área se encuentra condicionado y supeditado a su iniciativa y motivación personal.

Siempre está el apoyo [por parte del equipo directivo], lo que pasa es que ellos te dicen en todo que sí, pero después uno tiene que estar pidiendo por ejemplo, el profe, no sé, de Matemáticas que me preste a un niño, porque necesitamos practicar [para 
una jornada de debate interescolar]; entonces yo siempre trato de mandar los horarios a los alumnos, para que ellos hablen con los profesores, pero aquí los canales de comunicación en algún momento se pierden, entonces finalmente, si uno no lo hace, si uno no se mueve, ellos [directivos] no, porque también tienen mucho trabajo (Docente establecimiento municipal, Santiago).

En la relación de los equipos directivos con los docentes, ya sea en esta autonomía o en este abandono, se puede reafirmar la evidencia previa respecto del excesivo énfasis y concentración puesto en la asignatura y en el docente de Historia (Comisión Formación Ciudadana, 2004; Mardones, 2015), así como el sesgo disciplinario y epistemológico en el trabajo de formación ciudadana, en que el resto de las asignaturas no se perciben como relevantes.

Tal como se señaló en el apartado teórico, el trabajo formativo escolar de la ciudadanía supone la conjunción coherente de tres ámbitos: currículo, relaciones intrainstitución, y relaciones entre esta y la comunidad externa. La Ley No 20.911 al exigir un plan integral de desarrollo de la formación ciudadana a cada institución, empuja a que el liderazgo y la docencia consideren los tres contextos evocados en forma coherente. Como se constata, este camino está en sus inicios, y el pasado y sus anclas aún predominan. En este sentido, el desencuentro de visiones entre directivos y docentes y la concentración de responsabilidades en la asignatura de Historia y Ciencias Sociales revela una problemática aún por resolverse. Esto, pues seguir el camino pedagógico — que avala la evidencia de la investigación internacional del área- como propulsor del conocimiento, actitudes y participación de los estudiantes, implica un trabajo mancomunado entre diferentes actores y asignaturas y un liderazgo con visión global e integradora.

\subsection{Liderazgo e instancias de representación estudiantil}

La constatación del estudio respecto de un ámbito formativo clave de las actitudes y habilidades ciudadanas — como son las instancias de participación y representación como centros de estudiantes y directivas de curso- es triple: su existencia es generalizada, 
ocupan un lugar importante en los PFC, y, al mismo tiempo, en los establecimientos estudiados no tienen mayor significado para los estudiantes y se evidencia un potencial formativo subaprovechado en este importante ámbito de formación. En general, se observa que no existe una visión o un proyecto coherente para la promoción de dichas instancias: su quehacer se limita a actividades como organización de eventos y dependen del interés de cada generación o grupo de estudiantes. Las instancias referidas a las que se agrega en ciertos casos el Consejo Escolar, son valoradas y promovidas tanto por directores como docentes, aun cuando se observa una reducción de la participación escolar en las elecciones del centro de estudiantes, que figuran como el único referente educativo de participación democrática.

Los niños van a votar y esperan el proceso de votación, y van ordenados, o sea, tampoco es un desorden, ellos van ordenados, pero a su turno, van, emiten su voto, y se van a la sala de clases, y les gusta participar, sobre todo a los más chiquititos, niños de séptimo, octavo, ¡les encanta! (Director establecimiento municipal, Copiapó).

Asimismo, la mayoría de los espacios de representación son de tipo formal y no deliberativo o de toma de decisiones, ratificando la evidencia recogida previamente (ACE, 2015b; PNUD, 2018). Aunque esto tiene excepciones, como testimonian los PFC que examinamos en la sección siguiente (ver Tabla 2).

El espacio intraescolar, tan evidentemente relacional en la esencia de su quehacer cotidiano - el encuentro y conversación permanente de dos generaciones- ofrece un potencial de especial riqueza para la práctica de habilidades, actitudes y valores de directa conexión con lo que a lo largo del artículo se ha distinguido como la dimensión cívica, es decir, política, de la formación ciudadana. Esto es, las relaciones y prácticas de deliberación, representación, influencia, negociación, procesamiento del conflicto, y construcción de acuerdos. Todas y cada una de ellas intrínsecas al funcionamiento del sistema político, e identificadas como claves de comunicar y de ser practicadas en la experiencia escolar por la corriente principal 
de la investigación y las evaluaciones internacionales del área (Kerr, 2015; Schulz, Ainley, Fraillon, Losito \& Agrusti, 2016). Sin embargo, no fue posible apreciar que las instancias de representación escolar pudieran garantizar experiencias significativas de participación, o bien, de valoración democráticas en las que hubiera un diseño o priorización de las relaciones aludidas desde el liderazgo y los docentes, produciéndose el subaprovechamiento de estos espacios. Con todo, hay excepciones y ejemplos contundentes del sentido contrario: en algunos casos se constató la participación de los estudiantes en el diseño de aspectos del reglamento escolar, modificaciones de estatutos, y coherente tratamiento de las elecciones de centro de alumnos (campaña, debates, foro, elecciones, voto secreto, libre e informado), lo que habla de la asignación de valor formativo trascendente y poder significativos para las instancias de representación bajo examen.

La voz de los estudiantes, sin embargo, de manera bastante transversal, es crítica respecto de estas instancias y destaca la carencia de significación de los centros de estudiantes y su baja incidencia como canal efectivo de participación.

La mitad del curso fue a votar, y al final terminaron yendo al baño, terminaron yéndose a jugar a alguna parte, pero no fueron a votar; y de los pocos que votaron, había muchos que decían "ya, mi amiga va a votar por este, entonces yo también voto por este" y no reflexionan, que cuando uno vota por el centro de estudiantes, aquí es un beneficio propio (...) entonces, al final termina siendo una elección súper superficial (Estudiantes establecimiento municipal, Temuco).

No todos los testimonios refieren una experiencia tan desprovista de significado para la formación ciudadana: debe tenerse presente el entusiasmo en la votación por el centro de alumnos por parte de los cursos menores mencionados en la cita anterior, así como los expresados en los PFC (ver Tabla 2). Entre ambos lados de este chiaroscuro debe visualizarse el gran potencial que, al parecer, hoy no se está explotando por parte del liderazgo, que valora estos espacios, pero que en general (en la limitada muestra de nuestro estudio), 
los visualiza en forma restringida y sin la especificidad formativa de relaciones y prácticas directamente relacionadas con la vida política.

3.5. PFC: heterogeneidad, valor potencial y percepción de bajo impacto

Finalmente, se observa un alto grado de discrecionalidad en los establecimientos a la hora de definir no solo sus visiones, sino también sus acciones respecto de la formación ciudadana. Un espectro educacional marcado por la prevalencia de proyectos públicos y privados, muchos de ellos confesionales, de orientación humanista-científica o técnico profesional, junto con el carácter generalista y abierto del PFC mandatado por el Mineduc produce una gran diversidad y heterogeneidad de lo que cada establecimiento entiende y, por tanto, forma como ciudadanos. Debido a esto, la identidad del establecimiento juega un importante rol a la hora de definir el tipo de ciudadanía que inspira su acción formativa.

Si bien los directivos de los establecimientos estudiados valoran y perciben el PFC como un programa importante, de acuerdo con sus palabras, este no ha implicado un cambio significativo en el quehacer institucional. Para ellos, el plan ha sido un aporte en la línea de visualizar y reconocer la importancia de la temática y hacer necesaria una reflexión y acciones de diseño y planificación que articulan un conjunto de iniciativas de formación ciudadana que ya existían en los establecimientos, pero que se encontraban dispersas y fragmentadas.

La Tabla 2 ofrece una visión sinóptica de tres PFC de establecimientos de la muestra del estudio: su heterogeneidad en términos de volumen de acciones consideradas, foco, y profundidad y coherencia formativa hace ver que no corresponde generalizar en cuanto al carácter de este nuevo instrumento y su impacto en el quehacer de las instituciones. 


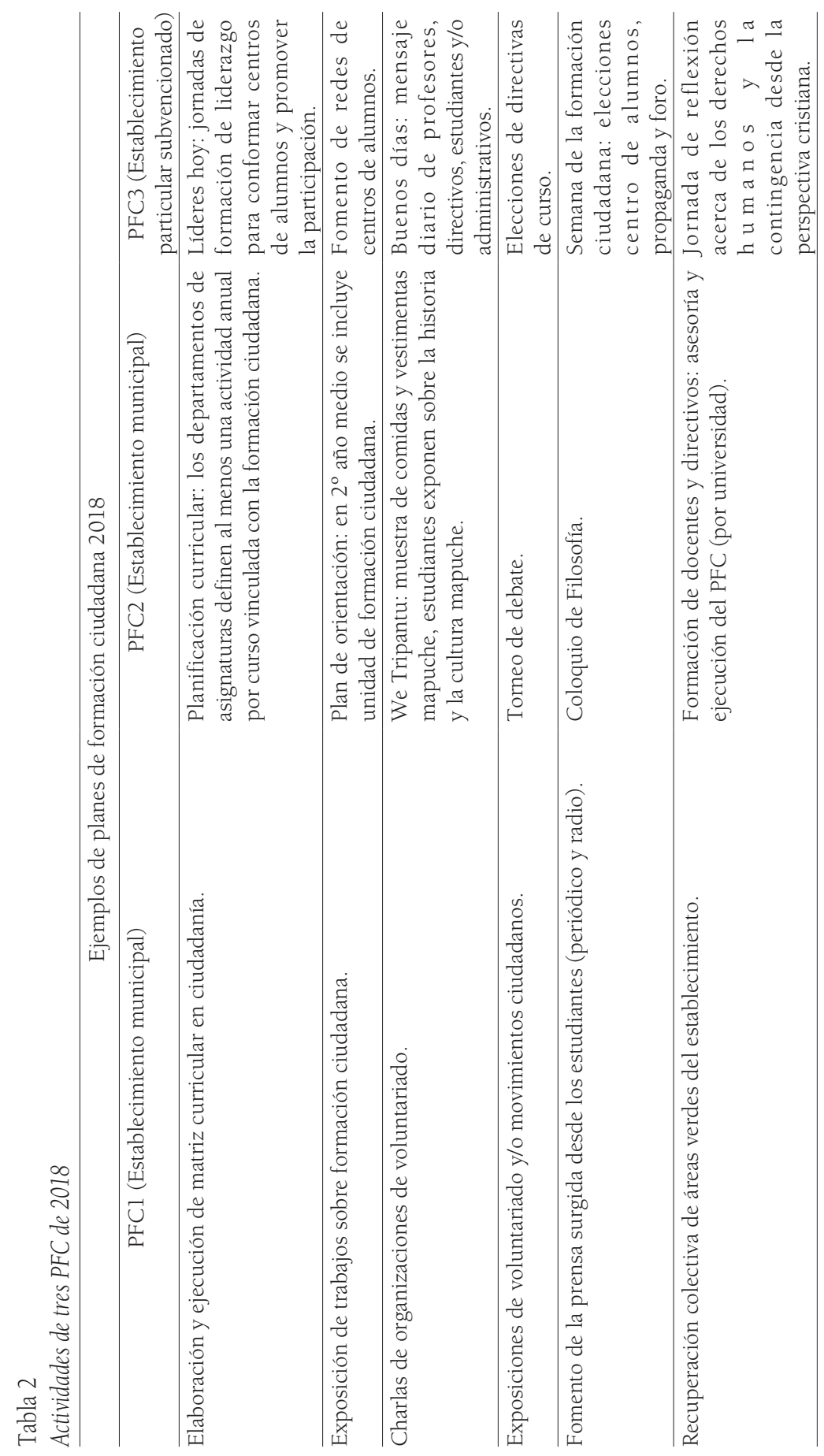




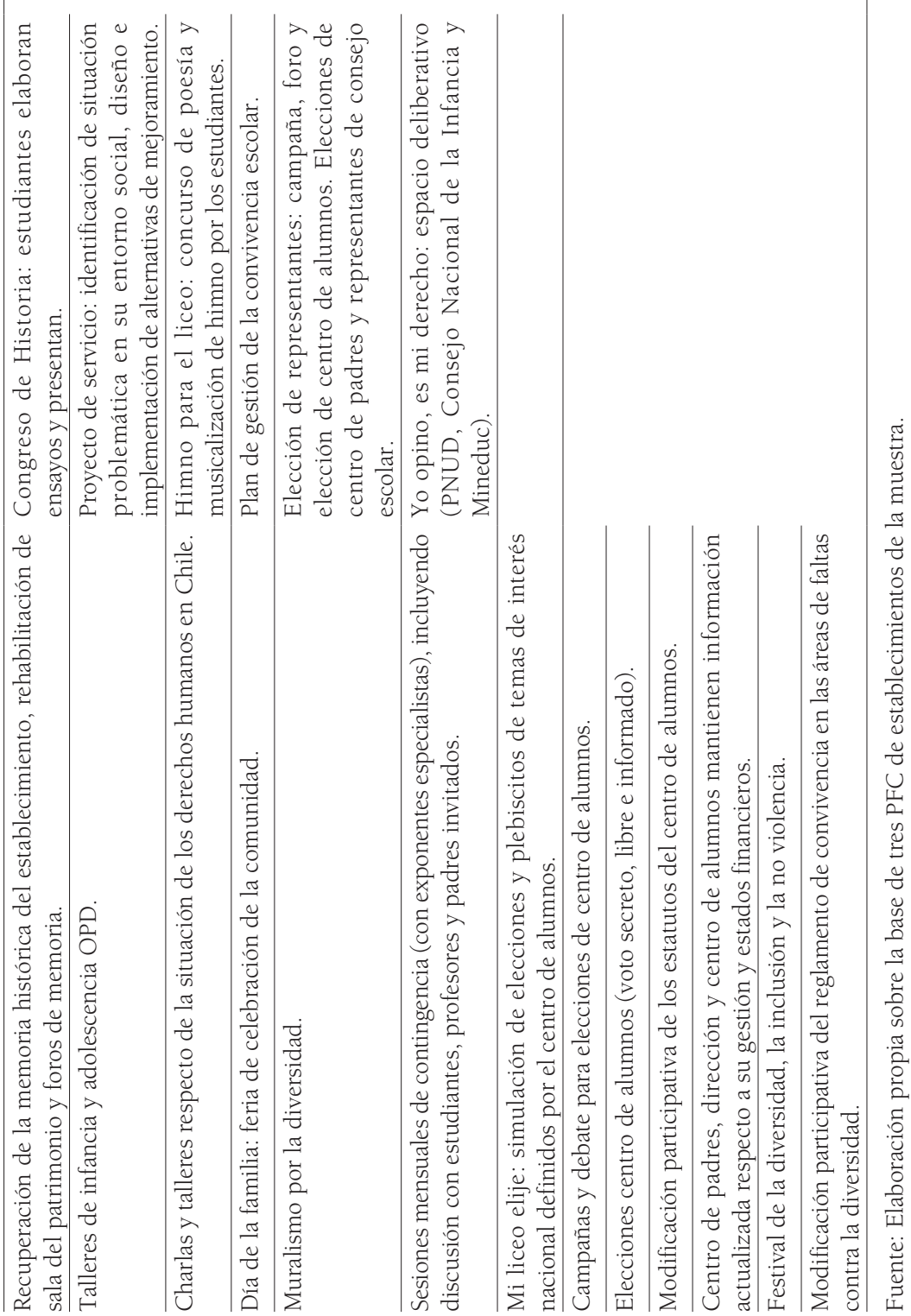


Hay una evidente escala de completitud, ambición y profundidad formativa en las actividades propuestas de los tres PFC, la que va desde el PFC 1 (20 actividades que 'hacen sistema') al PFC 3 (solo seis actividades, tres de las cuales se refieren al centro de alumnos), con el PFC 2, en una situación intermedia (12 actividades, con diferentes focos). Si se considera el plan más completo y ambicioso —el PFC 1-, se observa que el rango de los focos formativos que abordan sus 20 actividades abarcan el currículo, el centro de alumnos, la contingencia política, temáticas de derechos humanos y medio ambiente, nuevas formas de participación política (rendición de cuentas y transparencia) y proyectos de servicio a la comunidad. Si se recuerda el modelo de tres ámbitos clave para la oferta coherente de formación ciudadana (currículo, relaciones participación-representación intrainstitución, relaciones con comunidad externa local, y macro), los PFC 1 y 2 abordan (con diferencias en su riqueza) cada una de ellas, mientras que el PFC 3 se centra solo en una de ellas (participación vía centro de alumnos), y toca tangencialmente otra (relación con comunidad externa macro, al proponer discutir temas de derechos humanos y contingencia), e ignora el currículo.

Por cierto que junto con las diferencias marcadas entre los PFC, no debe perderse de vista la distancia que media entre un documento que plasma un plan o proyecto y su realización. Así como también es altamente relevante la perspectiva que interroga acerca de cómo son producidos los PFC y su grado de apropiación por parte del cuerpo docente y demás actores de un establecimiento. Se recogió también en las entrevistas que sus modos de generación e implementación varían también considerablemente en grados de participación, involucramiento y sistematicidad. En términos generales, lo que se obtuvo de las entrevistas es que para los directores el plan no ha supuesto mayores cambios, y lo que los directivos definen como una sistematización de iniciativas de formación ciudadana corresponde, muchas veces, a un listado de actividades sin mayor ambición formativa ni sistematicidad (como ilustra el PFC 3 en la Tabla 2).

Cabe mencionar que los estudiantes destacan la falta de iniciativas intraescolares para fortalecer el vínculo con la comunidad extraescolar, la participación y el compromiso social. Destaca en ellos 
el uso del término "burbuja", transversal a las tres dependencias, para describir la manera en que se vive la experiencia escolar, protegida y relativamente aislada de las condiciones sociales externas.

\section{Discusión e implicancias}

En la información recogida se observó que un indicador de formación ciudadana más alto tiene relación con el nivel de consolidación de la identidad del proyecto educativo, y esto último, a su vez, evidencia una marcada influencia de las prácticas y trayectoria de los directores. Esto es importante, ya que el componente identitario y cohesivo ha sido destacado como un factor fundamental para el ejercicio de la ciudadanía (Flanagan, 2003). En este contexto, el líder educativo es un actor clave en la articulación del entramado microcultural e identitario que favorece la pertenencia de los miembros a una comunidad, lo que constituye una robusta base relacional y socioafectiva para el trabajo formativo de las capacidades de la ciudadanía.

Por otra parte, se observa un alto grado de discrecionalidad en el actuar de los establecimientos a la hora de definir sus visiones y acciones respecto de la formación ciudadana. Si bien en el discurso de los directivos existe una marcada preocupación por esta área, en la práctica se observa que no hay mayor distinción entre educación para la convivencia (u otros cercanos, interacción) y educación para la ciudadanía (otros lejanos, sistema político), y que se tiende a priorizar la formación valórica, sin distinguirse suficientemente los requisitos formativos específicos del ciudadano o la participación política. Esto genera una gran diversidad y heterogeneidad acerca de lo que cada establecimiento entiende y, por tanto, forma como ciudadanos. Se constata que el foco en la alfabetización política no ha resultado prioritario, quedando estas temáticas relegadas a los contenidos revisados y provistos exclusivamente en las clases de Historia y Ciencias Sociales. Con ello, en el discurso de los jóvenes la ciudadanía resulta un constructo lejano, normativo y perteneciente al mundo adulto, configurándose en su visión una ciudadanía sin apropiación.

Si bien existen instancias de representación de los profesores y los estudiantes, estas no tienen un peso sustantivo dentro del 
quehacer institucional, y tampoco existe en general (aunque hay excepciones importantes, incluso en el pequeño universo de los casos estudiados), un proyecto formativamente ambicioso y coherente para la promoción de dichos espacios, recayendo en la motivación y visión de los distintos actores de cada proyecto institucional.

Asimismo, se detectan prácticas de liderazgo pedagógico en el sentido de que se trata de directores que facilitan, proveen autonomía y condiciones favorables para que los docentes ejerzan sus labores educativas. Si bien esta última visión es predominante, para algunos docentes esto no es experimentado como algo positivo, sino como un abandono o falta de apoyo desde el equipo directivo hacia su labor en la transmisión de contenidos de formación ciudadana.

Desde los directivos, el PFC ha sido un aporte en la línea de visualizar y ordenar las iniciativas referentes al tema dentro de cada institución, con las ventajas que esto conlleva en términos de reflexividad del conjunto del cuerpo docente y directivo al respecto. Sin embargo, al existir una visión del área que tiende a confundir en general la convivencia, la formación valórica y la ciudadanía, se diluye el foco referido a conceptos, habilidades y actitudes específicamente atingentes a la participación ciudadana en tanto política. Con esto, lo que los directivos definen como una sistematización de iniciativas se trata muchas veces de un listado de actividades con poca coherencia y ambición formativa referida a este foco. Desde los estudiantes, en tanto, no existe mayor conocimiento sobre el PFC y no logran conectar ciertas actividades que han realizado en sus centros educativos con tareas propias y constitutivas del mismo.

Con todo lo anterior, se concluye que posiblemente debido a las limitaciones de los criterios de selección de la muestra, en los establecimientos seleccionados no se logró identificar necesariamente un conjunto de "buenas" prácticas de liderazgo en relación con la formación ciudadana, sino más bien un conjunto de prácticas desde las que se pueden extraer tanto fortalezas como nudos problemáticos. De esta forma, desde el análisis se observa que el liderazgo educativo tiene un papel relevante, aunque indirecto, en la formación ciudadana en sus dimensiones civiles, relacionadas con el desarrollo de identidad y sentido de pertenencia. 
Por otra parte, se identifican aspectos problemáticos relacionados con el excesivo énfasis y concentración de responsabilidades puestos en la asignatura y en el docente de Historia en términos de la educación ciudadana, así como la prevalencia de la formación valórica por sobre la alfabetización política, lo que tiene consecuencias en la manera en que el PFC y las instancias de representación escolar son definidas, valoradas y aprovechadas.

\section{Referencias}

Agencia de Calidad de la Educación, ACE. (2015a). Desarrollo personal y social: otros indicadores de calidad educativa (documento metodológico). Recuperado de http://archivos.agenciaeducacion.cl/Desarrollo_ personal_social_OIC_25_11.pdf

Agencia de Calidad de la Educación, ACE. (2015b). Los indicadores de desarrollo personal y social en los establecimientos educacionales chilenos: una primera mirada. Recuperado de http://archivos.agenciaeducacion. cl/estudios/Estudio_Indicadores_desarrollo_personal_social_en_ establecimientos_chilenos.pdf

Agencia de Calidad de la Educación, ACE. (2016). El liderazgo del equipo directivo en enseñanza básica y su relación con indicadores de desarrollo personal y social de su establecimiento. Recuperado de http://www. agenciaeducacion.cl/wp-content/uploads/2016/02/Liderazgo_ directivo_y_relacion_indicadores_desarrollo_personal_social.pdf

Agencia de Calidad de la Educación, ACE. (2017). Estudio internacional de educación cívica y formación ciudadana. Presentación nacional de resultados. Recuperado de https://www.agenciaeducacion.cl/estudios/ estudios-internacionales/iccs/

Amnå, E. (2012). How is civic engagement developed over time? Emerging answers from a multidisciplinary field. Journal of Adolescence, 35(3), 611-627. https://doi.org/10.1016/j.adolescence.2012.04.011

Amnå, E., Ekström, M., Kerr, M., \& Stattin, H. (2009). Political socialization and human agency: The Development of civic engagement from adolescence to adulthood. Statsvetenskaplig Tidskrift, 111(1). Recuperado de http://journals.lub.lu.se/index.php/st/article/view/8141

Bascopé, M., Cox, C., y Lira, R. (2015). Tipos de ciudadano en los currículos del autoritarismo y la democracia. En C. Cox y J. C. Castillo (Eds.), Aprendizaje de la ciudadanía: contextos, experiencias y resultados (pp. 245-282). Santiago de Chile: Ediciones Universidad Católica de Chile. 
Bonhomme, M., Cox, C., Tham, M., y Lira, R. (2015). Educación ciudadana escolar de Chile "en acto": prácticas docentes y expectativas de participación política de estudiantes. En C. Cox y J. C. Castillo (Eds.), Aprendizaje de la ciudadanía: contextos, experiencias y resultados (pp. 373-425). Santiago de Chile: Ediciones Universidad Católica de Chile.

Campbell, D. E. (2008). Voice in the classroom: How an open classroom climate fosters political engagement among adolescents. Political Behavior, 30(4), 437-454. https://doi.org/10.1007/s11109-008-9063-z

Castillo, J. C., Miranda, D., Bonhomme, M., Cox, C., \& Bascopé, M. (2014). Mitigating the political participation gap from the school: The roles of civic knowledge and classroom climate. Journal of Youth Studies, 18(1), 16-35. https://doi.org/10.1080/13676261.2014.933199

Comisión Formación Ciudadana. (2004). Informe Comisión Formación Ciudadana. Santiago de Chile: Ministerio de Educación.

Consejo Asesor Presidencial Contra los Conflictos de Interés, el Tráfico de Influencias y la Corrupción. (2015). Informe. Recuperado de http:// consejoanticorrupcion.cl/wp-content/uploads/2015/06/2015.06.05consejo_anticorrupcion.pdf

Cox, C. (Ed.). (2003). Políticas educacionales en el cambio de siglo: la reforma del sistema escolar de Chile. Santiago de Chile: Editorial Universitaria.

Cox, C., Bascopé, M., Castillo, J. C., y Miranda, D. (2015). Educación ciudadana en América Latina: prioridades de los currículos escolares. En C. Cox y J. C. Castillo (Eds.), Aprendizaje de la ciudadanía: contextos, experiencias y resultados (pp. 321-372). Santiago de Chile: Ediciones Universidad Católica de Chile.

Cox, C., Bascopé, M., Castillo, J. C., Miranda, D., y Bonhomme, M. (2014). Educación ciudadana en América Latina: prioridades de los currículos escolares (No. 14). Ginebra: Unesco-IBE.

Cox, C. y García, C. (2015). Objetivos y contenidos de la formación ciudadana escolar en Chile 1996-2013: tres currículos comparados. En C. Cox y J. C. Castillo (Eds.), Aprendizaje de la ciudadanía: contextos, experiencias y resultados (pp. 283-320). Santiago de Chile: Ediciones Universidad Católica de Chile.

Day, C., Sammons, P., Hopkins, D., Harris, A., Leithwood, K., Gu, Q., ... Kington, A. (2009). The impact of school leadership on pupil outcomes. Final report to DSCF. Nottingham: Department of Children, Families $\&$ Schools/National College of School Leadership.

Dudley, R. L. \& Gitelson, A. R. (2002). Political literacy, civic education, and civic engagement: A return to political socialization? Applied 
Developmental Science, 6(4), 175-182. https://doi.org/10.1207/ S1532480XADS0604_3

Flanagan, C. (2003). Developmental roots of political engagement. PS: Political Science and Politics, 36(2), 257-261. https://doi.org/10.1017/ s104909650300218x

Flanagan, C. \& Levine, P. (2015). Involucramiento cívico y la transición a la adultez. En C. Cox y J. C. Castillo (Eds.), Aprendizaje de la ciudadanía: contextos, experiencias y resultados (pp. 75-110). Santiago de Chile: Ediciones Universidad Católica de Chile.

Flessa, J., Bramwell, D., Fernández, M., \& Weinstein, J. (2018). School leadership in Latin America 2000-2016. Educational Management Administration \& Leadership, 46(2), 182-206. https://doi. org/10.1177/1741143217717277

Friedkin, N. E. (2004). Social cohesion. Annual Review of Sociology, 30(1), 409425. https://doi.org/10.1146/annurev.soc.30.012703.110625

Hallinger, P. \& Heck, R. H. (2010). Collaborative leadership and school improvement: Understanding the impact on school capacity and student learning. School Leadership E Management, 30(2), 95-110. https://doi.org/10.1080/13632431003663214

Horn, A. y Marfán, J. (2010). Relación entre liderazgo educativo y desempeño escolar: revisión de la investigación en Chile. Psicoperspectivas. Individuo y Sociedad, 9(2), 82-104. https://doi.org/10.5027/psicoperspectivasvol9-issue2-fulltext-116

Isac, M. M., Maslowski, R., Creemers, B., \& Werf, G. van der. (2014). The contribution of schooling to secondary-school students' citizenship outcomes across countries. School Effectiveness and School Improvement, 25(1), 29-63. https://doi.org/10.1080/09243453.2012.751035

Jara Ibarra, C., Sánchez, M., y Cox, C. (2019). Liderazgo educativo y el desarrollo de competencias ciudadanas en el contexto escolar. Recuperado de http:// cedle.cl/wp-content/uploads/2019/05/cuaderno-17.pdf

Joignant, A., Morales, M., \& Fuentes, C. (Eds.). (2017). Malaise in representation in Latin American. Chile, Argentina, and Uruguay. Recuperado de http:// www.palgrave.com/br/book/9781137599872

Kerr, D. (2015). Ciudadanía a nivel nacional, regional e internacional: una revisión de enfoques, investigaciones y debates. En C. Cox y J. C. Castillo (Eds.), Aprendizaje de la ciudadanía: contextos, experiencias y resultados (pp. 111-143). Santiago de Chile: Ediciones Universidad Católica de Chile. 
380 LIDERAZGO EDUCATIVO Y FORMACIÓN CIUDADANA: VISIONES Y PRÁCTICAS DE LOS ACTORES - C. Jara, M. Sánchez y C. Cox

Leithwood, K., Harris, A., \& Hopkins, D. (2008). Seven strong claims about successful school leadership. School Leadership \& Management, 28(1), 27-42. https://doi.org/10.1080/13632430701800060

Leithwood, K. \& Jantzi, D. (2000). The effects of different sources of leadership on student engagement in school. En K. Riley \& K. Louis (Eds.), Leadership for change and school reform: International perspectives (pp. 112-129). London, New York: Routledge/Falmer.

Leithwood, K., Sammons, P., Anderson, S., \& Wahlstrom, K. (2004). A review of research. How leadership influences student learning. The Wallace Foundation. Ontario: Center for Applied Research an Educational Improvement/Ontario Institute for School Leadership.

Ley No 20.911 Crea El Plan De Formación Ciudadana Para Los Establecimientos Educacionales Reconocidos Por El Estado. Diario Oficial de la República de Chile, Santiago, Chile, 2 de abril de 2016. Recuperado de http://bcn.cl/lv0oy

Mardones, R. (2015). El paradigma de la educación ciudadana en Chile: una política pública inconclusa. En C. Cox y J. C. Castillo (Eds.), Aprendizaje de la ciudadanía: contextos, experiencias y resultados (pp. 145-174). Santiago de Chile: Ediciones Universidad Católica de Chile.

Mardones, R., Cox, C., Farías, A., y García, C. (2014). Currículos comparados, percepciones docentes y formación de profesores para la formación ciudadana: tendencias y proposiciones de mejoramiento. En Centro de Políticas Públicas UC (Ed.), Propuestas para Chile concurso políticas públicas (pp. 215-245). Santiago de Chile: Pontificia Universidad Católica de Chile.

Marks, H. M. \& Printy, S. M. (2003). Principal leadership and school performance: An integration of transformational and instructional leadership. Educational Administration Quarterly, 39(3), 370-397. https://doi.org/10.1177/0013161x03253412

Martínez, M. L., Silva, C., Morandé, M., y Canales, L. (2010). Los jóvenes ciudadanos: reflexiones para una política de formación ciudadana juvenil. Última Década, 18(32), 105-188. https://doi.org/10.4067/ S0718-22362010000100006

McDevitt, M. \& Kiousis, S. (2006). Deliberative learning: An evaluative approach to interactive civic education. Communication Education, 55(3), 247-264. https://doi.org/10.1080/03634520600748557

Ministerio de Educación de Chile, Mineduc. (2015). Marco para la buena dirección y el liderazgo escolar. Recuperado de http://liderazgoescolar. mineduc.cl/wp-content/uploads/sites/55/2016/04/MBDLE_2015.pdf 
Ministerio de Educación de Chile, Mineduc. (2004). Informe Comisión Formación Ciudadana. Recuperado de https://es.slideshare.net/ annyhen/informe-formacin-ciudadana-en-chile

Mitra, D., Serriere, S., \& Stoicovy, D. (2012). The role of leaders in enabling student voice. Management in Education, 26(3), 104-112. https://doi. org/10.1177/0892020612445678

Programa de las Naciones Unidas para el Desarrollo, PNUD. (2018). Estudio sobre la puesta en marcha del Plan de Formación Ciudadana. Recuperado de http://www.ciudadaniayescuela.cl/wp-content/uploads/2018/04/ Estudio-puesta-en-marcha-del-Plan-de-Formaci\%C3\%B3nCiudadana.pdf

QSR International Pty Ltd. (2014). Nvivo [software] (Version 10).

Robinson, V., Hohepa, M., \& Lloyd, C. (2009). School leadership and student outcomes. Identifying what works and why: Best evidence synthesis iteration (BES). Wellington: Ministry of Education.

Schulz, W., Ainley, J., Fraillon, J., Kerr, D., \& Losito, B. (2010). ICCS 2009 international report: Civic knowledge, attitudes, and engagement among lower secondary school students in 38 countries. Amsterdam: International Association for the Evaluation of Educational Achievement.

Schulz, W., Ainley, J., Fraillon, J., Losito, B., \& Agrusti, G. (2016). IEA International Civic and Citizenship Education Study 2016. Assessment Framework. Recuperado de http://www.springer.com/us/ book/9783319393568

Vaillant, D. (2011). Improving and supporting principals' leadership in Latin America. En T. Townsend \& J. MacBeath (Eds.), International handbook of leadership for learning (pp. 571-585). https://doi.org/10.1007/97894-007-1350-5_32

Waters, T., Marzano, R. J., \& McNulty, B. (2003). Balanced leadership: What 30 years of research tells us about the effect of leadership on student achievement. Recuperado de http://files.eric.ed.gov/fulltext/ ED481972.pdf

Whyte, J. (1999). Political socialization in a divided society: The case of Northern Ireland. En M. Yates \& J. Youniss (Eds.), Roots of civic identity: International perspectives on community service and activism in youth (pp. 156-177). Cambridge: Cambridge University Press. 